\title{
Transferable skills of undergraduates of sciences and arts at Taibah University, El-Ula Branch, Saudi Arabia
}

\author{
Salem Albalawi *, Samy Zalat, Somia El-Akkad, Zenab Deghash \& Safaa Ramadan \\ Faculty of Science \& Arts, Taibah University, El-Ula Branch, Saudi Arabia.
}

\begin{abstract}
Transferable skills constitute a principle element in the curriculum of all educational programmes. Some human skills are transferred with birth in genetic factors, but these skills need to be discovered and developed: university teaching programmes are the main source to teach and develop these skills. This study is designed to explore the skills abilities of undergraduates as they see themselves and their abilities. Students showed great variation in skills abilities in the seven studied skills; they showed high ability for teamwork, communication, personal and development skills. Second-year students seemed more skilful than those of the first year. Women were highly skilled relative to men, the difference mostly being how wide the gap is between women and men in the seven categories of skills. When we compared Arts and Science students in these skills, there were significant differences between them, mainly in that science students were more skilful in information technology and numerical skills. These data reflect the impact of social traditions on skill development rather than as a part of the teaching process, which means that effort should be put into creating courses which will develop the skills of students.
\end{abstract}

Keywords: Education, skill classification, gender, grade, curriculum.

\section{Introduction}

Transferable skills are difficult to define and the term has some ambiguity. Field (1998) defined them as skills that are by their nature used in different contexts, learnt in education and applied to employment. The formal definition (Drummond 1998) is those skills that are central to occupational competence in all sectors and all levels, including project management, leadership, communication, working in teams and problem solving. Transferable skills are "portable skills" that you deliberately take with you to other life experiences. They can be divided into three subsets including working with people, things and information/data.

Transferable skills can be acquired through informal life experiences or formal education and training, and can be transferred from one setting to another. Although the specific subject matter of academic disciplines is often a means for developing or refining them, their application is not dependent on mastery of an academic discipline. Transferable skills are interdisciplinary abilities involving many areas of human development (e.g., cognitive, affective, social, psychological, and moral development). Transferable skills are very important for mental and personal development of individuals. We are born with some skills, but others need to be discovered and further developed after birth (Morgil \& Ural 2006). A number of authors have developed generic frameworks which may be applied sequentially throughout an individual's life cycle: foundation-level skills such as basic knowledge or personal traits; practical skills, which include occupational knowledge or personal attributes such as communication or empathy; and advanced capabilities comprising generic competencies or 'meta-abilities' such as leadership or teamworking (Butcher et al. 1997).

Throughout life we gain new skills and competencies. Some are learnt in formal situations - in school, vocational training courses, college or university. Others we learn through our work. Many come from less formal settings - home, our hobbies or social groups to which we belong. In Saudi Arabia, one of the most conservative of cultures, society and 
social activities play an important role in determining the skills acquired by students. The community is a mixture of different cultures and educational backgrounds, including Egyptian, Jordanian, Syrian, Tunisian, Lebanese, and many Southeast Asian countries. The education system is no exception: most staff are foreigners with different educational backgrounds. The two factors of living in a culture accommodating such a wide range of nationalities, yet with its own traditions and practices, play major roles in determining to great extent the skills acquired.

This paper focuses on identifying the skills of students beginning their university degrees, using a self-evaluation questionnaire that supports people in evaluating their own possible competencies, enabling them to discover their own competencies. This makes them 'visible', allowing us to make use of them in designing the subjects and programmes of education, especially in newly established institutions such as the Faculty of Science \& Arts of Taibah University. We feel that starting by looking at all the skills that individuals possess may open up new possibilities and opportunities which might never previously have been considered. This is the impression we gained from students during this study.

Therefore this paper is aimed at assessing students' perceptions of the skills they have acquired during their previous life experiences and education; identifying the key skills needed to be taught in the subsequent years of their degrees; finding out the main differences in ability in skill categories by gender, year group, academic department, sub-faculty and any interactions among categories.

\section{Materials \& Methods}

The study was conducted using a questionnaire distributed to 319 students of all departments of the Faculty of Science \& Arts, Taibah University, El-Ula branch, Saudi Arabia. They represent all departments of the Faculty: Arabic $(28 \hat{\jmath}, 61 q)$, Islamic Studies $(14 \hat{\jmath}, 28$ 우),

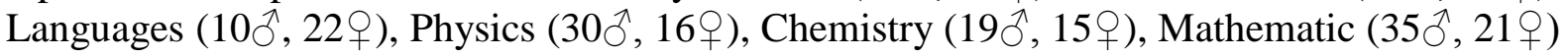
and Biology (20ㅇ). The transferable-skills questionnaire was developed specifically for use in this study. Skills were classified and the questionnaire developed in the light of the literature (Morgil \& Ural 2006; Clarkeburn et al. 2000; Howe 2002). With a total of 59 items, the questionnaire asked about seven main skill groups and various sub-groups:

(a) searching skills (finding sources, using knowledge and sources effectively, organizing information, applying methods and procedures effectively, designing experiments, data quality assurance, the ability to observe and take notes, the ability to differentiate between theories and ideas, the ability to apply knowledge, the ability to extract main ideas from articles, making decisions based on data, making conclusions and summaries, and respecting intellectual property rights);

(b) teamwork and communication skills (working in groups, respecting the ideas of others, motivating people, collaborating and making group decisions, working positively with others, persuading others, respecting others, empathy and handling meetings);

(c) written and oral presentation skills (expressing ideas clearly, preparing written and oral presentations, speaking in public comfortably, preparing effective reports, and presenting reports);

(d) personal development skills (being responsible, critical thinking, time management, constructive criticism, being creative, working with discipline, being self-confident, the ability to imagine, the ability to make decisions, the ability to work under stress, negotiation, self-control, respect for the surrounding environment, planning meetings, working independently); 
(e) information technology and numerical skills (using computers, preparing reports on a computer, using presentation materials, using emails, using statistical software, presenting graphical data, using simple calculations, conducting effective presentations);

(f) linguistic skills (reading and understanding old and recent literature, using the correct language to express ideas, proper scientific writing);

(g) psychomotor skills (using physical abilities e.g. music, drawings, handcrafts etc.). The answers to the 59 questions were scored on a four-point scale: zero (no ability), 1 (weak ability), 2 (noticeable ability), 3 (high ability).

Because there were multiple answers per student, we used linear mixed models with Poisson errors, with both fixed and random predictors. The analysis by SPSS 18 used generalized estimating equations, and the significance of each factor was assessed by the change in deviance upon its deletion from the model, distributed as $\chi^{2}$. The predictors were a set of factors (skill categories, gender, year group, semester, faculty, department) that were 'fixed', and one (individual student) that is a 'random' factor. The response variable is the score, the reply given by the student to each question: these are integer numbers from 0 to 3 , and therefore we used Poisson errors.

\section{Results}

There were no significant differences in skill scores of students among the seven departments, all with very similar average scores. There were significant differences among skill categories $\left(\chi^{2}=460.6, \mathrm{df}=6, \mathrm{p}<0.05\right)$. Students showed high mean scores for teamwork and personal development skills relative to the other categories (Fig 1).

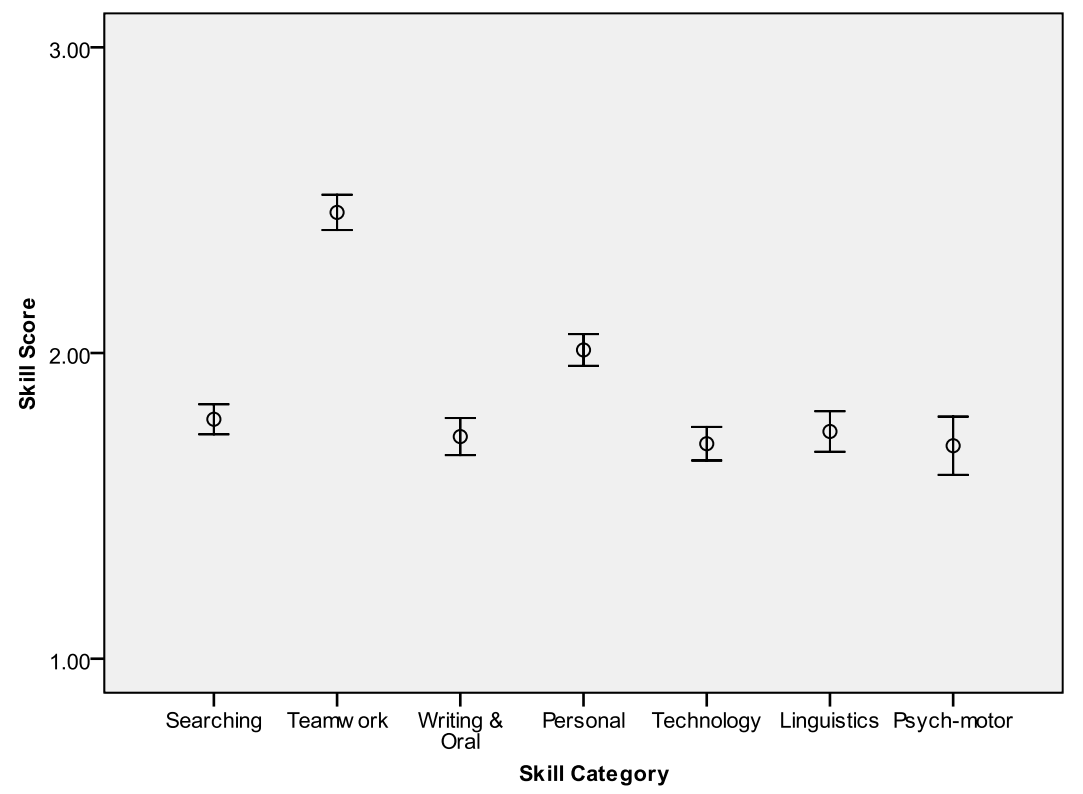

Figure 1: Mean values ( \pm s.e.) for seven categories of skills as recorded by the Arts \& Science students from various departments of Taibah University at El-Ula.

We compared second-year students with different first-year backgrounds, i.e. those who had spent two semesters studying in a preparatory year and one semester in their departments (Languages, Mathematics, Physics, Chemistry \& Biology), as against those who had spent all three semesters studying in their departments (Arabic \& Islamic Studies). The data showed a highly significant difference in the mean scores of these types of student $\left(\chi^{2}=8.9\right.$, 
df $=1, \mathrm{p}<0.05$ : Fig 2), with students of Arabic and Islamic studies on average showing lower skill scores than those of Science and Language students.

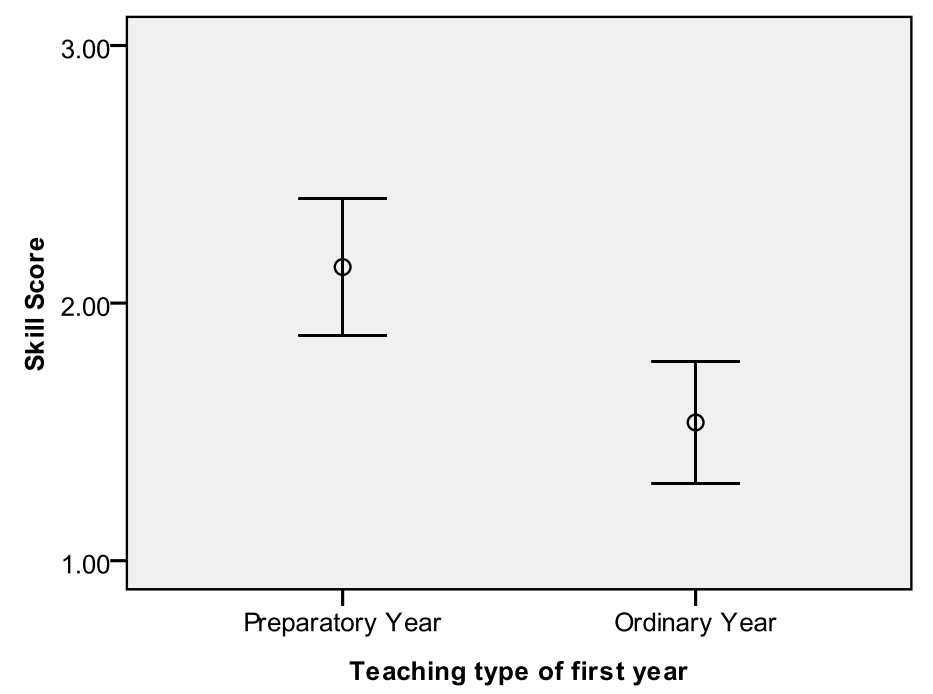

Figure 2: Mean skill scores of second-year students who had a preparatory year (Science and Languages) and those who had had an ordinary year in their programme (Arabic and Islamic Studies).

Comparing first-year students (who had had only one teaching semester) with second-year students (who had had three semesters) also showed an overall significant difference in mean skill score $\left(\chi^{2}=4.2, \mathrm{df}=1, \mathrm{p}<0.05\right)$, with second-year students having higher scores (Fig 3 ).

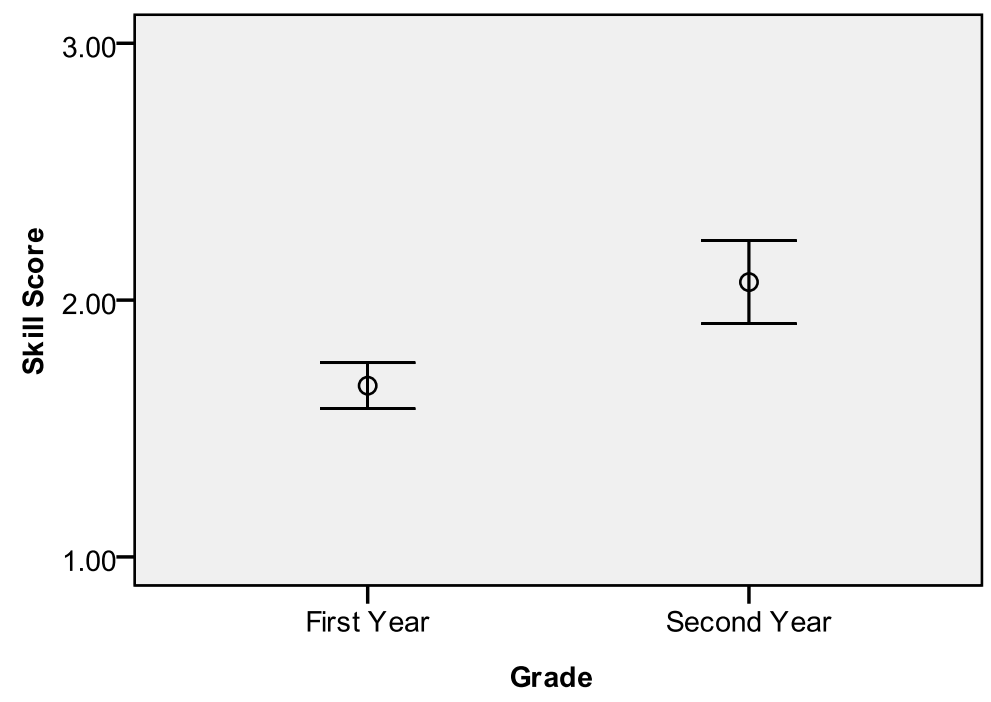

Figure 3: Mean skill scores of first-year students (with one semester's teaching) and secondyear students (with three semesters).

As expected, women showed much a higher mean skill score than men, a difference that was highly significant $\left(\chi^{2}=18.8, \mathrm{df}=1, \mathrm{p}<0.05\right.$ : Fig 4). 


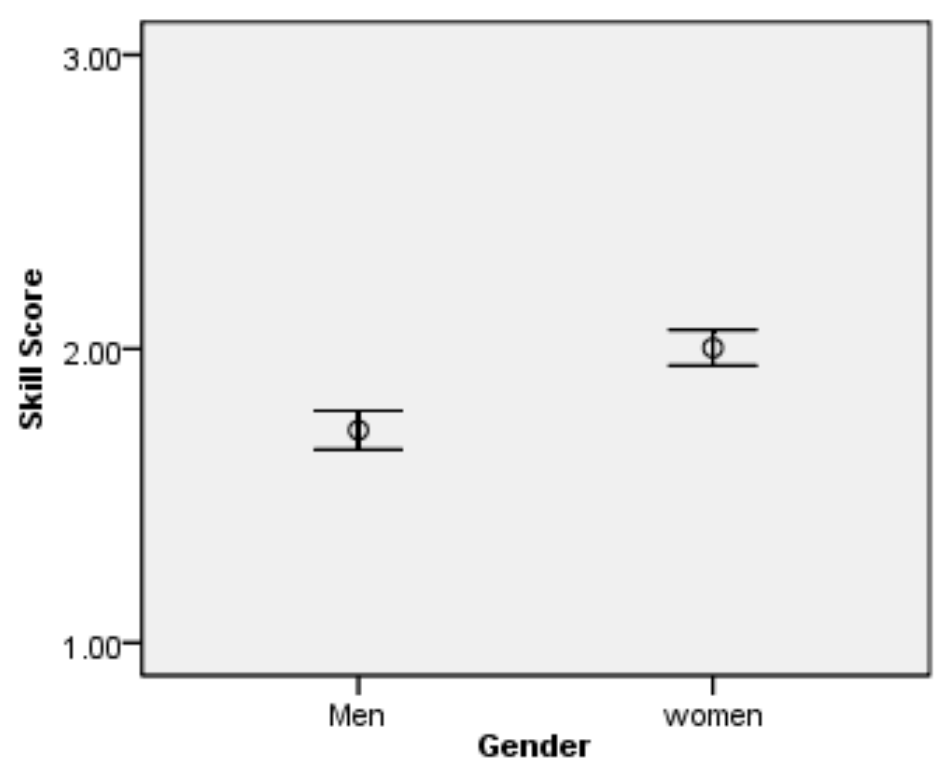

Figure 4: Mean skill scores of male and female students.

We tested the interaction between the field of study (Science versus Arts), and the seven skills categories, and it was statistically significant $\left(\chi^{2}=12.9\right.$, df $=6, p<0.05$ : Fig 5). Science students showed a different set of skill abilities from Arts students: the main difference seems to be in information technology and numerical skills, where Science students score higher than arts students (see Fig 5). Arts students showed slightly higher scores in personal and linguistic skills than Science students.

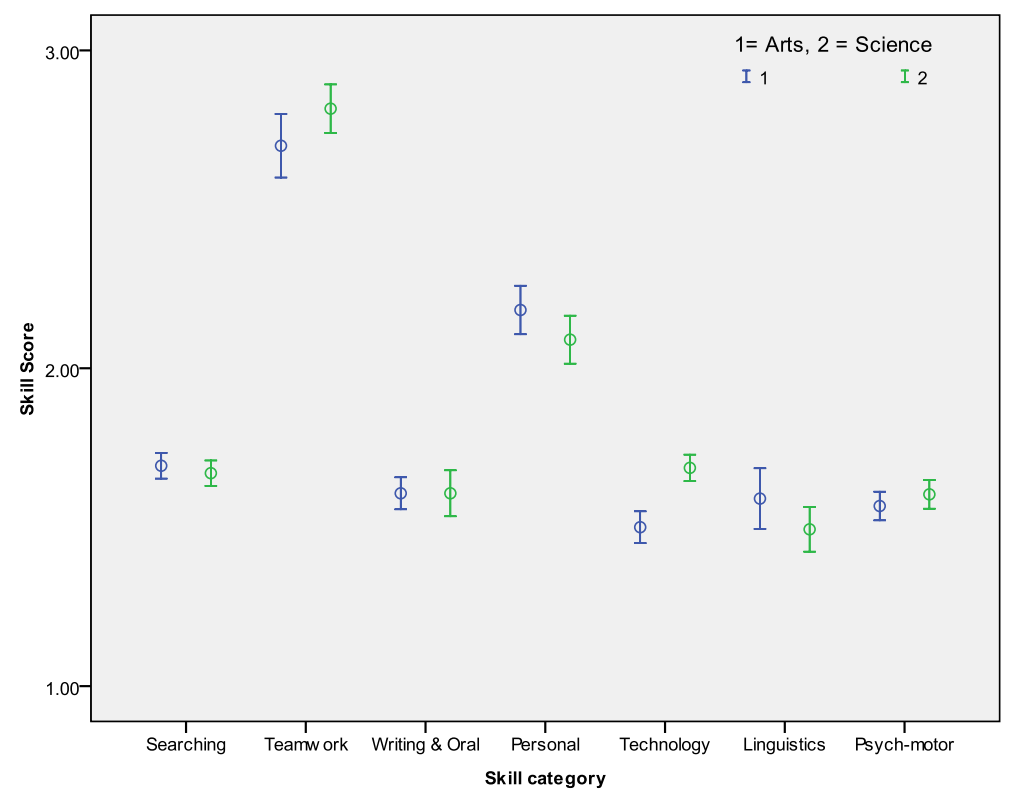

Figure 5: Mean skill scores of the seven skill categories for students with different teaching backgrounds (Arts and Science). 
The interaction between skill category and gender also showed a very highly significant effect $\left(\chi^{2}=24.2\right.$, df $=6, p<0.05$ : Fig 6), mostly reflecting the width of the gap between men and women, with women having consistently higher mean scores across all categories.

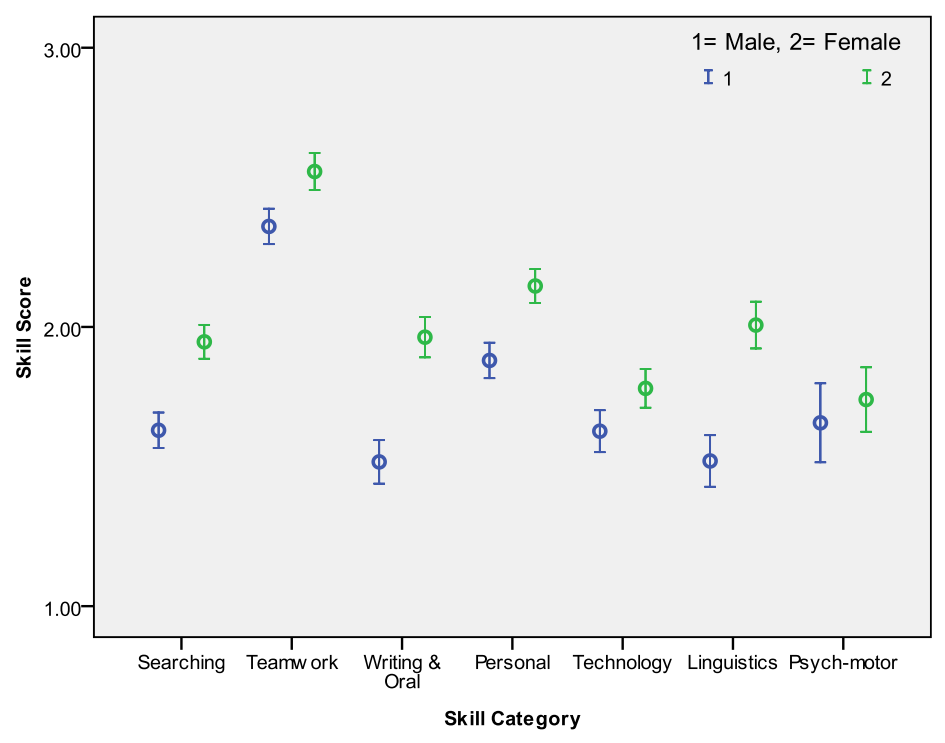

Figure 6: Interaction between gender and the scores of the seven categories of skills.

\section{Discussion}

Skills concepts have long been influential in UK higher education (e.g. Payne 2000), most recently with the recommendation that learning outcomes be formulated in terms of key skills (Dearing 1997) and the inclusion of subject and generic skills in course benchmarking statements (QAA 2002). Transferability is a key feature of the concept of skills, and has been the main focus of challenges to the value of skills approaches (e.g. Whitston 1998). Certain criteria have been described explicitly as skills. Critical thinking, for example, was defined as 'a propensity and skill to engage in an activity with reflective scepticism' (Halonen 1995). Writing and use of language are also often approached as generic skills that can be developed independently of what is being written about, especially when the focus is on grammar, punctuation and referencing conventions. Structuring is also sometimes approached in a skills-oriented way.

It has been suggested that when developing a new programme, skills criteria can be identified at the initial planning stages, and hence modules can be tailored to teach and assess the desired skills (Borthwick 2002). In this light, this study looked at what kind of skills students of the Faculty of Science \& Arts of Taibah University thought they had. The data showed great variation in perceived ability in the seven skill categories. Students thought they had high abilities in teamwork, communication, personal and development skills, but less ability in searching, writing and oral presentations, information technology and numerical skills, linguistic and psycho-motor skills. Burke et al. (2005) referred to some skills (such as communication, application of number, information technology and self reliance) as 'transferable common core', and 'key', depending upon the particular initiative, organization or sector under consideration. The skills the students perceived seem to be acquired from less formal settings - from home, or from commonly practised social activities. Saudi society implements a culture of responsibility and encourages young people in activities usually either unavailable or reserved for older ages in other cultures, e.g. driving cars, taking care of 
the old or young family members, participating in meeting and serving guests in a polite and organized way, and many other activities. We believe these practices help students to gain skills early on, including teamworking and personal skills. Since Saudi society is very conservative, separation between men and women is very rigid (at least in the El-Ula governorate), which we think pushes young people to use communication facilities such as mobile phones in a very professional way: communication technology equipment is freely available and affordable. Teamwork and personal skills are valued by parents and the whole society.

Some authors classify skills into soft and hard skills: soft skills are the non-technical skills necessary for success in the workplace, such as interpersonal and human relations, professional social, organizational management, time management, critical thinking and problem solving. Hard skills are not as "transferable" as soft skills. They are more closely associated with a specific discipline of study, or career, and are the technical skills necessary for success in the workplace, also referred to as content-specific skills. They include pharmaceutical, biological, architectural, computing, mathematical, therapeutic, teaching and graphic design skills. In this study, student perceptions of their searching and psychomotor skills are relatively low, and seem to be classified as soft skills: however, these contain technical abilities as well and need to be taught in the curriculum. The data support this idea: second-year students think themselves more skilful than first-year students in all types of skill, even in searching and psychomotor skills, which may reflect the impact of teaching in the curriculum.

The results suggest that women consider themselves much more skilful than men, a major difference in all seven skill categories. Mumford (1987) put an emphasis on the idea of students interacting with their environment by constantly reviewing experiences and linking fresh material to prior knowledge. In the Saudi environment, women are very keen on education: going to school and university is one of their major daily outdoor activities, and families are also very keen for women to get an education and to join not only official programmes within governmental education bodies, but also all types of vocational education - workshops, training courses, seminars, etc. Furthermore, women have more time and opportunity than men in Saudi society. Usually young men are responsible for performing many duties, and it is obvious that attendance of male students is very low compared to that of women. Women are always given a ride to school or university by a male family member (husband, father, brother), and are not allowed to be left on their own. This means that women spend most of their time in education benefitting from teaching programmes and activities. In contrast, male students have responsibilities within their families, and most have to help in daily activities of life. As a consequence, attendance rates of male students are often very low. When asked about attendance, male students reply that they have to do urgent family duties. Within the home, normal women's duties are usually supported and performed by employed staff such as housekeepers, i.e. cleaning, cooking and other household activities are usually done by someone else. This situation perhaps enhances the chances for women to acquire skills. Even for communication and interpersonal skills, in which one might expect men to be more skilful than women, again the data show that Saudi women consider themselves to be more skilful than men. This is perhaps due to the fact that women practise more or less the same level of social activities as men, but there is no mix in these activities.

The issue of skills development has been at the forefront of the Higher Education agenda for some time (CVCP 1998). Over the last five years, there has been an increasing awareness of the need for a post-16 education experience that provides breadth, flexibility and an understanding of the need for progressive skills development as students move into higher education (Burke et al. 2005). 
There has been considerable interest recently in the teaching of skills to undergraduate students. However, existing methods for collecting data on how much, where and when students are taught and assessed skills have often been shown to be time-consuming and ineffective (Fraser et al. 2007). British universities have been required to offer graduate students services in professional development and training in 'transferrable skills'. The professional development services typically run for two weekends in a year. In Europe, France has initiated various reforms, one of which established 'doctoral schools' offering an array of workshops, seminars and courses for candidates and institutional cooperative ventures. Everyone seems to have a different view about how graduates should develop skills and how they should most effectively demonstrate a set of skills. Some believe a structured approach should be used. Others argue for a more open and flexible approach that embraces work-integrated learning and cognitive apprenticeships.

In our work, the staff members of Arts and Science departments were given two ways of delivering skills, either via special courses or within the teaching of technical courses. There was clearly an overwhelming response in favour of the latter manner, meaning there was greater support for the integration of skills into the curriculum. This strongly suggests that departments should aim to convey skills that are more than purely vocational in nature. It is obvious that we should concentrate on finding the best ways of delivering skills to our students, paying attention to the softer skills and reducing the quantity of knowledge we deliver in our courses within the Science \& Arts and departments in Taibah University. This study not only highlights the importance of getting the skills agenda right, but somewhat flies in the face of many traditional approaches to higher education curricula which focus upon the importance of knowledge and technical or 'hard' skills, and underemphasize the softer or process aspects of skills (Silver 1991).

We used a questionnaire to measure student perceptions of their skills, but clearly we need to develop a way of assessing the reality of their skills abilities, i.e. are they really obtaining these skills? It seems clear that we should develop more robust mechanisms for measuring and assessing skills development throughout the undergraduate experience. We hope that future studies will continue using samples from the same range of undergraduate programmes across all years, which will allow a comparison between them. Without such a direction, student learning may be left purely to chance: we need to find more creative ways of communicating, developing and measuring skills on an ongoing basis.

\section{Acknowledgements}

We give our sincere thanks to the Deanship of Scientific Research, Taibah University and the Scientific Committee for their kind support for this proposal and for securing the funds to complete this work. We thank also the staff members of the Faculty of Science \& Arts, Taibah University, El-Ula Branch for their useful and fruitful discussion; to the students of the Faculty of Science \& Arts of El-Ula for their interest and enthusiasm for the study. Special thanks to Dr Francis Gilbert (Nottingham University, UK) for helping us to analyze the data.

\section{References}

Borthwick F (2002) Skills Plus: Audit of the undergraduate higher national programmes in the construction group at Liverpool John Moores University. Available at: http://www.cebe.heacademy.ac.uk/learning/ casestudies/case_pdf/Borthwickf.pdf

Burke V, Jones J \& Doherty M (2005) Analysing student perceptions of transferable skills via undergraduate degree programmes. Active Learning in Higher Education, 6(2): 132-144.

Butcher D, Harvey P \& Atkinson S (1997) Developing businesses through developing individuals. Bedford: Cranfield University.

CVCP (1998) Skills development in Higher Education. London: CVCP.

Dearing R (1997) Higher Education in the learning society: report of the National Committee of Enquiry into Higher Education. London: HMSO. 
Drummond M (1998) Personal transferable skills in Higher Education: the problems of implementing good practice, Quality Assurance in Education, 6(1): 19-22

Field R (1998) Embedding transferrable skills in the adult education curriculum, Adults and Learning, 9(5): 1214.

Fraser GA, Crook AC \& Park JR (2007) A tool for mapping research skills in undergraduate curricula. Bioscience Education, 9: 1-12.

Halonen JS (1995). Demystifying critical thinking. Teaching of Psychology, 22(1): 75-81.

Morgil I \& Ural E (2006) The effect of computer-supported education and internet usage on pre-service chemistry teachers' transferable skills in active learning environments. Journal of Turkish Science Education. 3(2): 35-38.

Mumford A (1987) Using reality in management development. Management Education \& Development, 18(3): 223-243.

Payne J (2000) The unbearable lightness of skill: the changing meaning of skill in UK policy discourses and some implications for education and training. Journal of Educational Policy, 15(3): 353-369.

Quality Assurance Agency for Higher Education (2002) Benchmarking academic standards: subject statements (phase 2). www.qaa.ac.uk/crntwork/benchmark/phase2consult_textonly.htm.

Silver M (1991) Competent to manage. London: Routledge.

Whitston K (1998) Key skills and curriculum reform. Studies in Higher Education, 23: 307-319.

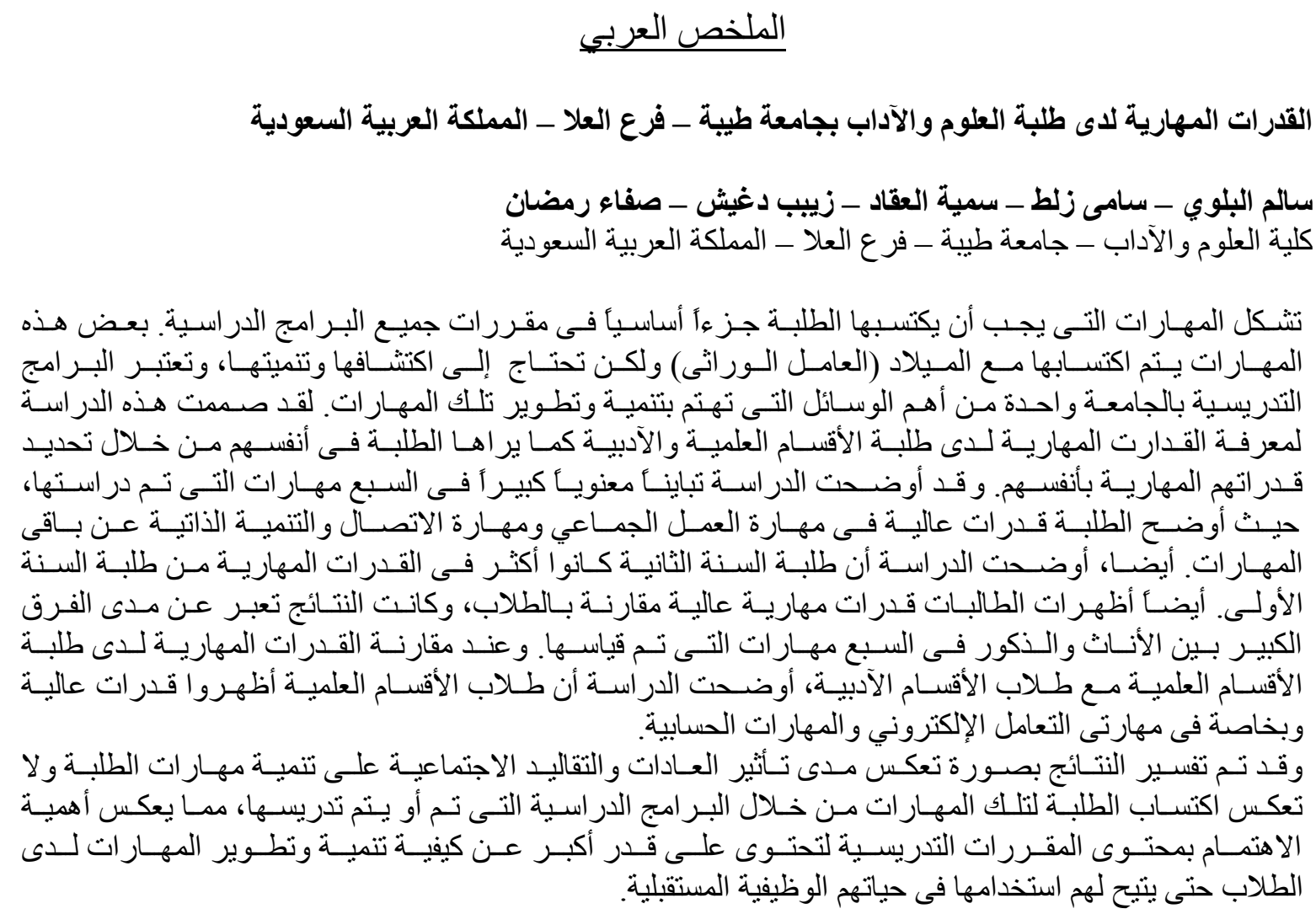

